\title{
Micro-foundations of congestion and pricing: A game theory perspective
}

\author{
David Levinson * \\ Department of Civil Engineering, University of Minnesota, 500 Pillsbury Drive SE Minneapolis, MN 55455, United States
}

Received 8 October 2003; received in revised form 26 July 2004; accepted 22 February 2005

\begin{abstract}
This paper develops congestion theory and congestion pricing theory from its micro-foundations, the interaction of two or more vehicles. Using game theory, with a two-player game it is shown that the emergence of congestion depends on the players' relative valuations of early arrival, late arrival, and journey delay. Congestion pricing can be used as a cooperation mechanism to minimize total costs (if returned to the players). The analysis is then extended to the case of the three-player game, which illustrates congestion as a negative externality imposed on players who do not themselves contribute to it.
\end{abstract}

(C) 2005 Elsevier Ltd. All rights reserved.

Keywords: Game theory; Congestion; Queueing; Traffic flow; Congestion pricing; Road pricing; Value pricing

\section{Introduction}

Congestion, one of the most frustrating problems in both freight and passenger transportation, vexes policy-makers, while an understanding of the technical foundations of congestion among policy analysts remains weak. Walters (1961) and Mohring (1970) apply microeconomic theory to congestion, but assume aggregate demand functions and take no account of variation in time or schedule delay (i.e. the journey time is the same for all travelers in the peak). Vickery (1969) and

\footnotetext{
* Tel.: +1 612625 6354; fax: +1 6126267750 .

E-mail address: levin031@umn.edu
} 
Arnott et al. (1990, 1993, 1998) among others relax that uniform journey time assumption in the bottleneck model, which takes into account time variation as a factor in congestion, and allows travelers to trade-off journey time for schedule delay (see Lindsey and Verhoef (2000) for a summary). These models are extremely useful, but still consider congestion as a product of many travelers.

Schelling (1978) argues that macroscopic phenomena should be examined from their microfoundations, the behaviors of individuals. This paper takes that approach, aiming to build the simplest possible congestion model that reflects real phenomena of schedule delays as well as negative congestion externalities. The paper treats congestion as, at its core, a relatively simple phenomenon with a relatively simple solution. This paper considers congestion as comprising multiple interacting players. The departure time decisions of one commuter affect the journey delay and arrival times experienced by other commuters, leading to interactions and possible gains to all players by cooperating. This paper uses the term journey delay, or travel time in excess of uncongested times, to contrast with schedule delay which refers to the difference in time of departure or arrival compared with preferred conditions. (Other decisions: shifts in mode, route, location, or destination also affect demand of other commuters, but are not included here for clarity of presentation). Game theory, developed by Von Neumann and Morgenstern (1944), presents an analytic approach to explain the choices of multiple actors (agents) in conflict with each other with scope for cooperation, where the payoffs are interdependent (Hargreaves-Heap et al., 1995; Osborne and Rubinstein, 1994; Taylor, 1987). This is distinct from decision theory, where the opponents are states of nature and are passive (Rapoport, 1970). Games are generally classified by the number of players (the games described here are two and three player) and whether the game is zero sum or not. Non-zero sum games engender benefits from cooperation that are absent in zero sum games. Pricing can be a seen as a mechanism to achieve the benefits of cooperation.

The application of game theory requires acceptance of certain assumptions about the behavior of actors and their level of knowledge. First, it is assumed that actors are instrumentally rational. Actors who are instrumentally rational express preferences (which are ordered consistently and obey the property of transitivity) and act to best satisfy those preferences. Here it is assumed that travelers minimize total costs (the sum of congestion journey delay penalties, schedule delay penalties [arriving early or late] and prices).

Second, it is assumed that there is common knowledge of rationality (CKR). Common knowledge of rationality assumes that each actor is instrumentally rational, and that each actor knows that each other actor is instrumentally rational, and that each actor knows that each other actor knows, and so on.

Third, it is assumed that there is a consistent alignment of beliefs (CAB). Each actor, given the same information and circumstances, will make the same decision-no actor should be surprised by what another actor does.

Last, it is assumed all players know the rules of the game, including all possible actions and the payoffs of each for every player. This assumption of perfect knowledge, which runs through traditional route choice and congestion pricing models, is strong, and is realistic only in a simple, highly structured game. This assumption is used for expository purposes here, so that the model does not become too complex. Clearly it would be desirable to extend the model to deal with imperfect information, as discussed in the conclusions, though the extent to which that changes 
the basic results is unknown. In particular, this assumption is more valid for recurring congestion rather than non-recurring congestion (due to incidents, construction, weather, etc.).

The congestion game described is a variant of the famous "prisoners' dilemma" game. A Nash equilibrium is a set of strategies such that no player can improve by changing strategy given that other players keep their strategies fixed. This corresponds very closely with the independently developed Wardrop (1952) User Equilibrium principle used in route choice, and the insight gained from Knight's (1924) illustration of the equal costs of two used routes. Players' choose a Nash (user) equilibrium, but if both players were to cooperate, their payoff collectively is higher. A repeated prisoner's dilemma game may lead to cooperation either implicitly by self-interested players choosing an efficient strategy (e.g., tit-for-tat) or through development of a cooperation-enforcement mechanism. Cooperation in indefinitely repeated games may generate the highest collective payoff (the system optimal solution) (Axelrod, 1984). However because congestion in general is a large multi-driver phenomenon, we expect that cooperation cannot be achieved simply through repetition. A jointly agreed, externally imposed enforcement mechanism would reduce transactions (or bargaining) costs and may achieve a system optimal solution.

Game theory has been applied to a number of issues involving transportation such as to help understand airport landing fees (Littlechild and Thompson, 1977), fare evasion and compliance (Jankowski, 1991), truck weight limits (Hildebrand et al., 1990), merging behavior on freeways (Kita et al., 2001), how jurisdictions choose to finance their roads (Levinson, 1999, 2000), aviation (Hansen and Wenbei, 2001), and the political acceptability of road pricing (Marini and Marcucci, 2003). This paper applies game theory to develop the micro-foundations of congestion, and extends the case to congestion pricing. While roadway congestion is normally thought of as a phenomenon involving hundreds or thousands of vehicles, congestion at its most basic, it simply involves two. Examples of significant, time consuming congestion at other transportation facilities that in fact only involve a few (or two) vehicles include ships seeking to use canal locks, airplanes seeking to use airport runways or gates, and trains competing for a single track. Another example is two cars seeking to use a one-car ferryboat to cross a river. In the above situations, those two may want to use a facility that can only accommodate one at any given time, forcing the other to wait. If there were no penalty for arriving early or late, the individuals might coordinate their actions to arrive at different times. However, if there is an advantage to arrive at a particular time (the cost of being early or late exceeds the cost of journey delay), congestion may be a natural consequence.

The next section develops a two-player game theoretic congestion model. This is followed by the introduction of pricing to improve the outcome. The subsequent section extends the model to a three-player game. The paper concludes with discussion of applicability of the model and potential extensions.

\section{Two-player congestion game}

It should be possible to describe congestion from first principles, using simple vehicle interactions. In its simplest form, this game theoretic model requires three variables and some assumptions. First, we need to define a penalty for early arrival $(E)$ and for late arrival $(L)$. There is also a penalty for suffering journey delay $(D)$. There are two players, or vehicles. Each (vehicle 1 and 
vehicle 2) has the option of departing early, departing on-time or departing late. If both vehicles depart (from their origin) at the same time, there will be congestion and one vehicle will arrive (at their destination) in the next time slot.

- If both individuals depart early (a strategy pair we denote as ee), one will arrive early and one will suffer journey delay but arrive on-time. We can say that each individual has a $50 \%$ chance of being early or suffering journey delay.

- If both individuals depart on-time (strategy oo), one will arrive on-time and one will suffer journey delay and arrive late. Each individual has a 50\% chance of suffering journey delay and being late.

- If both individuals depart late (strategy $l l$ ), one will arrive late and one will suffer journey delay and arrive very late. Each individual has a 50\% chance of suffering journey delay and being very late.

We can compute expected values for each of the nine strategy pairs, shown in Table 1 . The equilibrium solution clearly depends on the values of $E, L$, and $D$. However, the assumption that $E<D<L$, which was obtained by empirical studies such as Small (1982) and assumed in most theoretical analyses (e.g., Arnott et al. (1990)), is important to consider. This paper tests cases that both adhere to and violate the assumption. Payoffs are shown here as costs, so users are trying to minimize the values in the cells. Total social costs for a strategy pair are the sum of the values of vehicles 1 and 2 . The equilibrium conditions for the various states are shown in Table 2. As the table shows, while the uncongested strategy pairs eo and oe adhere to $E<D<L$, the congested strategy pair oo does not, though it does satisfy $E=D=L$ as well as other plausible circumstances.

Table 1

Payoff matrix

\begin{tabular}{llll}
\hline Vehicle 1 & Vehicle 2 & & \\
\cline { 2 - 4 } & Early & On-time & Late \\
\hline Early & {$[0.5 *(E+D), 0.5 *(E+D)]$} & {$[E, 0]$} & {$[E, L]$} \\
On-time & {$[0, E]$} & {$[0.5 *(L+D), 0.5 *(L+D)]$} & {$[0, L]$} \\
Late & {$[L, E]$} & {$[L, 0]$} & {$[L+0.5 *(L+D), L+0.5 *(L+D)]$} \\
\hline
\end{tabular}

Note: [Vehicle 1 payoff, Vehicle 2 payoff].

Table 2

Equilibrium conditions

\begin{tabular}{lll}
\hline State & No pricing & With pricing \\
\hline ee & $E=0$ and $D=0$ & $E=0$ and $D=0$ \\
$e o, o e$ & $E \leqslant 0.5 *(L+D)$ and $E \leqslant L$ & $E \leqslant L$ \\
$e l, l e$ & $E=0$ and $L=0$ & $E=0$ and $L=0$ \\
oo & $D \leqslant L$ and $0.5 *(L+D) \leqslant E$ & $L \leqslant E$ and $D=0$ \\
ol,lo & $L \leqslant E$ and $L \leqslant D$ & $L \leqslant E$ \\
$l l$ & $L=0$ and $D=0$ & $L=0$ and $D=0$ \\
\hline
\end{tabular}

Note: Assume penalties $E, L, D \geqslant 0$. 
Table 3

Example 1 Payoff matrix

\begin{tabular}{llll}
\hline Vehicle 1 & Vehicle 2 & & Ln-time \\
\cline { 2 - 4 } & Early & On- & \\
\hline$E, D, L 1,2,3$ & {$[1.5,1.5]$} & {$[1,0]^{*}$} & {$[3,1]$} \\
Early & {$[0,1]^{*}$} & {$[2.5,2.5]$} & {$[0,3]$} \\
On-time & {$[3,1]$} & {$[3,0]$} & {$[5.5,5.5]$} \\
Late &
\end{tabular}

Note: ${ }^{*}$ indicates Nash equilibrium.

Table 4

Example 2 Payoff matrix

\begin{tabular}{llll}
\hline Vehicle 1 & Vehicle 2 & & \\
\cline { 2 - 4 } & Early & On-time & Late \\
\hline$E, D, L$ 3,1,4 & & & \\
Early & {$[2,2]$} & {$[3,0]$} & {$[3,4]$} \\
On-time & {$[0,3]$} & {$[2.5,2.5]^{*}$} & {$[0,4]$} \\
Late & {$[4,3]$} & {$[4,0]$} & {$[6.5,6.5]$} \\
\hline
\end{tabular}

Note: ${ }^{*}$ indicates Nash equilibrium.

In example 1 (Table 3), we assume that the early arrival penalty is one unit, the journey delay penalty is 2 units, and the late arrival penalty is three units $(E=1, D=2, L=3)$. In this case, the Nash equilibrium is one vehicle departing early and the other on-time. If vehicle 1 departs ontime, vehicle 2 can do no better than depart early, and vice versa. This does not result in congestion (Table 4).

In example $2(E=3, D=1, L=4)$, the equilibrium is the congested strategy pair $o o$. But now the total welfare ( -5 units) is worse than the socially optimal choice strategy pairs $e o$ and $o e$ with a welfare of -3 units.

Other scenarios are given in the appendix. Most of these scenarios do not result in a congested outcome. Many of the scenarios have multiple equilibria. Empirical investigation is necessary to determine the most plausible values; however in general for travel to work, we expect the late arrival penalty to be greater than the early arrival penalty, (as in examples 1 and 2).

\section{Two-player congestion pricing game}

The model can be extended to deal with congestion pricing as shown in Table 5. (Table 2 again summarizes the equilibrium conditions.) We need to add a pricing term to the cells on the diagonal where congestion occurs $\left(\tau_{\mathrm{e}}, \tau_{\mathrm{o}}, \tau_{1}\right.$ for early, on-time, and late departure prices, respectively). Congestion is contingent on demand, so congestion prices are similarly contingent. If congestion prices occur only when there is actual congestion, in 6 of the 9 off-diagonal positions no congestion prices are actually imposed, but are simply used as a threat. They constitute a threat in that they might (or might not) be paid dependent upon the actions of the other player. However, prices 
Table 5

Payoff matrix with congestion pricing

\begin{tabular}{llll}
\hline Vehicle 1 & Vehicle 2 & & \\
\cline { 2 - 4 } & Early & On-time & Late \\
\hline Early & {$\left[0.5 *(E+D)+\tau_{\mathrm{e}}, 0.5 *(E+D)+\tau_{\mathrm{e}}\right]$} & {$[E, 0]$} & {$[E, L]$} \\
On-time & {$[0, E]$} & {$\left[0.5 *(L+D)+\tau_{\mathrm{o}}\right.$,} & {$[0, L]$} \\
& & $\left.0.5 *(L+D)+\tau_{\mathrm{o}}\right]$ & \\
Late & {$[L, E]$} & {$[L, 0]$} & {$\left[L+0.5 *(L+D)+\tau_{1}, L\right.$} \\
& & & $\left.+0.5 *(L+D)+\tau_{1}\right]$ \\
Early & {$[0.5 *(E+D)+\operatorname{MAX}(0.5 *(D-E), 0)$,} & {$[E, 0]$} & {$[E, L]$} \\
& $0.5 *(E+D)+\operatorname{MAX}(0.5 *(D-E), 0)]$ & {$[(L+D),(L+D)]$} & {$[0, L]$} \\
On-time & {$[0, E]$} & {$[L, 0]$} & {$[2 L+D, 2 L+D]$} \\
Late & {$[L, E]$} &
\end{tabular}

do not belong in the off-diagonal position, since they should not be paid if both drivers behave in a manner to avoid congestion.

A question remains as to what the appropriate 'marginal social cost' (MSC) price is. Normally $\mathrm{MSC}=\partial \mathrm{TC} / \partial Q$. Yet if total cost (TC) is not a function, but discrete, we need to think in term of discrete mathematics, so that the appropriate basis for the price is the incremental social cost (ISC), where ISC $=\Delta \mathrm{TC} / \Delta Q$. The incremental private cost (IPC) is the additional amount each player pays in the absence of tolls to take the trip, their own travel time. The appropriate toll is $\tau=$ ISC - IPC.

The analysis is tricky, and perhaps subject to some interpretation. It is important to remember that the value of time in the vehicle is not the only cost that the congested vehicle suffers. The vehicle also bears a late penalty associated with journey delay.

Consider the scenario $l l$. What is the baseline? If the two players could somehow both play $l$ without congesting each other, each would have a payoff of $[L]$ (based on payoffs of $[L, E]$ or $[L, 0]$ for the first player, $[E, L]$ or $[0, L]$ for the second player). Two players playing $L$ without congesting each other would have a total cost $=2 L$. In case $l l$ the payoff (without tolls) is $[L+0.5 *(L+D), L+0.5 *(L+D)]$ (which gives a total cost $=3 L+D)$. The difference between the total costs in this case is $L+D$. The incremental private cost is the additional amount the individual pays compared to their baseline (which was $L$ ). So IPC $=L+0.5(L+D)-L=0.5(L+D)$. The toll is thus $\tau_{1}=\mathrm{ISC}-\mathrm{IPC}=(L+D)-0.5(L+D)=0.5(L+D)$.

The same argument can be used to determine $\tau_{\mathrm{o}}$. If both players could play on-time without congesting each other, their total payoff would be 0 . The total payoff in the case $o o$ is $L+D$. The incremental social cost is thus $L+D$. The incremental private cost is $0.5 *(L+D)$. The toll is thus $\tau_{\mathrm{o}}=\mathrm{ISC}-\mathrm{IPC}=0.5 *(L+D)$.

The case of journey delay because both vehicles depart early is more subtle, as here a vehicle is early or has a longer journey time, but it is only the additional journey time that is imposed by the other vehicle. Further, that second vehicle avoids the early arrival penalty. Applying the same logic as above to case $e e$, if each player could travel early without congesting the other, their individual payoff would be $E$, and the total cost would be $2 E$. The total cost in ee is actually $E+D$. Instead of being early, one player is now delayed. The incremental social cost is 
$(E+D)-2 E=D-E$. The incremental private cost is $0.5 *(D-E)$ (the individual moves from a payoff of $E$ to $0.5 *(E+D)$ ). The toll is thus $\tau_{\mathrm{e}}=\mathrm{ISC}-\mathrm{IPC}=\operatorname{MAX}(0.5 *(D-E), 0)$ (using the max function to avoid negative tolls). If the early arrival penalty were greater than the cost of delay, this implies a negative toll, which is illogical.

A vehicle does, however, have effects on the other vehicle, even in the absence of congestion. The presence of multiple vehicles and potential congestion led to pricing. The presence of prices may lead a vehicle to choose an alternative time of arriving at the bottleneck. This displacement in time (so a traveler now departs the bottleneck (arrives at their destination) early rather than on-time) may diminish utility. Table 6 summarizes the results from the three congested scenarios.

Returning to Example 2, but including congestion prices, gives us a revised Example 2, shown in Table 7. Now, rather than a congested equilibrium, we have two uncongested equilibria. Congestion prices will move either vehicle 1 or vehicle 2 to an early departure. This eliminates congestion (and so no congestion prices need be collected). Compared with the original example 2, one of the vehicles is now better off by 2.5 units, and the other is worse off by 0.5 units. Overall, the system is better by 2 units, though each player may not agree.

Table 8 summarizes the results from a number of scenarios. In cases where there is a Nash equilibrium that does not present the socially optimal solutions (e.g., $(3,1,4))$ in the unpriced case, we find that pricing will reduce costs and tend to increase the number of equilibria solutions. In cases (such as $(1,1,1)$ ) where there is a dysfunctional equilibrium along with several equilibria that have lower costs, we find pricing will move the equilibria to the lower cost solutions. Pricing eliminates congestion in the cases shown in Table 8 provided there is a non-zero delay penalty $(D>0)$, and sometimes even when $D=0$, e.g., $(3,0,4)$ (since journey delay is not the only cost of congestion, schedule delay matters as well).

Table 6

Summary of total costs, incremental social and private costs, and tolls for congesting cases

\begin{tabular}{llllll}
\hline $\begin{array}{l}\text { Departure } \\
\text { case }\end{array}$ & $\begin{array}{l}\mathrm{TC}(Q=2) \text { based } \\
\text { on Table } 1\end{array}$ & $\mathrm{TC}(Q=1)$ & $\begin{array}{l}\mathrm{ISC}=\Delta \mathrm{TC} \\
(\text { as } \Delta Q=1)\end{array}$ & IPC & $\tau$ \\
\hline$e e$ & $E+D$ & $2 E$ & $D-E$ & $0.5 *(D-E)$ & $\operatorname{MAX}(0.5 *(D-E), 0)$ \\
oo & $L+D$ & 0 & $L+D$ & $0.5 *(L+D)$ & $0.5 *(L+D)$ \\
$l l$ & $3 L+D$ & $2 L$ & $L+D$ & $0.5 *(L+D)$ & $0.5 *(L+D)$ \\
\hline
\end{tabular}

Table 7

Example 2, payoff matrix with congestion prices

\begin{tabular}{llll}
\hline Vehicle 1 & Vehicle 2 & & \\
\cline { 2 - 4 } & Early & On-time & Late \\
\hline$E, D, L 3,1,4$ & & & {$[3,4]$} \\
Early & {$[2,2]$} & {$[3,0]^{*}$} & {$[0,4]$} \\
On-time & {$[0,3]^{*}$} & {$[5,5]$} & {$[9,0]$} \\
Late & {$[4,3]$} & {$[9,9]$} & \\
\hline
\end{tabular}

Note: *indicates Nash equilibrium. 
Table 8

Two-player game summary table

\begin{tabular}{|c|c|c|c|c|c|c|}
\hline$E, D, L$ & $\begin{array}{l}\text { Number of } \\
\text { Nash equilibria } \\
\text { (unpriced) }\end{array}$ & $\begin{array}{l}\text { Solutions } \\
\text { (unpriced) }\end{array}$ & Total cost & $\begin{array}{l}\text { Minimum } \\
\text { total cost }\end{array}$ & $\begin{array}{l}\text { Number of Nash } \\
\text { equilibria (priced) }\end{array}$ & $\begin{array}{l}\text { Solutions } \\
\text { (priced) }\end{array}$ \\
\hline $0,0,0$ & 9 & all & 0 & 0 & 9 & All \\
\hline $0,1,0$ & 6 & eo,el,oe, ol,le,lo & 0 & 0 & 6 & eo,el,oe, ol,le,lo \\
\hline $0,0,1$ & 3 & $e e, e o, o e$ & 0 & 0 & 3 & $e e, e o, o e$ \\
\hline $0,1,1$ & 2 & oе,eo & 0 & 0 & 2 & oe,eo \\
\hline $1,0,0$ & 4 & $o o, o l, l o, l l$ & 0 & 0 & 4 & $o o, o l, l o, l l$ \\
\hline $1,1,0$ & 2 & $o l, l o$ & 0 & 0 & 2 & $o l, l o$ \\
\hline $1,0,1$ & 1 & $o o$ & 1 & 1 & 5 & eo, oe, oo, ol, lo \\
\hline $1,1,1$ & 5 & $e o, o e, o o, o l, l o$ & 1 or 2 & 1 & 4 & $e o, o e, o l, l o$ \\
\hline $1,2,3$ & 2 & $e o, o e$ & 1 & 1 & 2 & $e o, o e$ \\
\hline $3,1,4$ & 1 & $o o$ & 5 & 3 & 2 & $o e, e o$ \\
\hline $4,0,3$ & 1 & $o o$ & 3 & 3 & 3 & $o o, o l, l o$ \\
\hline $4,1,3$ & 1 & $o o$ & 4 & 3 & 2 & $o l, l o$ \\
\hline $3,0,4$ & 1 & $o o$ & 4 & 3 & 2 & $e o, o e$ \\
\hline
\end{tabular}

Note: Minimum total cost is the total cost in the cell with the lowest total cost.

\section{Three-player congestion pricing game}

The three-player game extends the two-player game described above. The three-player game gives us insight into congestion affecting a third party who does not behave "badly" by choosing to travel in a period with another traveler, but simply receives the spillover from other congesting traffic. The game is extended by one period (adding a very early penalty $(2 E)$ and strategy $(v)$ ), and the possibility exists (if all players enter the queue in the late period (strategy $l l l$ ) of one commuter facing a penalty of being really late $(2 L)$ (departing the queue two time slots after desired) and another commuter being super late $(3 L)$ (departing the queue three time slots after desired)).

There are 64 arrival patterns considering vehicle identification (which differ by which vehicle arrives in which slot) that reduce to 20 distinct arrival patterns (ignoring vehicle identification). However there are only 8 departure patterns, since only one departure can occur per time slot, but more than one arrival can occur in that time slot. Table 9 shows that with a queueing analysis maintaining first-in, first-out logic, the number of arrival patterns maps to considerably fewer departure patterns.

Expected journey delay at time $t, \varepsilon\left(d_{t}\right)$, here is measured as (assuming the service rate is defined as 1 vehicle per unit time):

$$
\varepsilon\left(d_{t}\right)=\left(Q_{t}+0.5\left(A_{t}-1\right)\right)
$$

The cost of journey delay is the expected journey delay multiplied by the delay penalty

$$
C\left(d_{t}\right)=\left(Q_{t}+0.5\left(A_{t}-1\right)\right) D
$$

where $D$ is the delay penalty; $Q_{t}$ the standing queue at time $t$ and $A_{t}$ is the arrivals at time $t$.

Schedule delay $\left(S_{i}\right)$ is the deviation from the time which a vehicle departs a queue and the desired, or on-time, period. It can thus be computed as 
Table 9

Three-player game arrival and departure patterns

\begin{tabular}{|c|c|c|c|}
\hline Arrival pattern & Frequency $(/ 64)$ & Departure patterns & Frequency $(/ 64)$ \\
\hline$v v v$ & 1 & veo & 16 \\
\hline voe & 3 & & \\
\hline vvo & 3 & & \\
\hline veo & 6 & & \\
\hline vee & 3 & & \\
\hline$v o l$ & 3 & vel & 9 \\
\hline vel & 6 & & \\
\hline voo & 3 & vol & 9 \\
\hline vol & 6 & & \\
\hline vll & 3 & $v l r$ & 3 \\
\hline eеe & 1 & eol & 16 \\
\hline$e е o$ & 3 & & \\
\hline eel & 3 & & \\
\hline eоo & 3 & & \\
\hline eol & 6 & & \\
\hline ell & 3 & elr & 3 \\
\hline ooo & 1 & olr & 7 \\
\hline ool & 3 & & \\
\hline oll & 3 & & \\
\hline$l l l$ & 1 & lrs & 1 \\
\hline
\end{tabular}

Note: $v$ indicates vehicles arrive/depart very early, $e$ early, $o$ on-time, $l$ late, $r$ really late, $s$ superlate.

$$
S_{i}=t_{\mathrm{a}}+d_{t}-t_{\mathrm{o}}
$$

where $d_{t}$ is the journey delay; $t_{\mathrm{a}}$ the time of arrival at back of queue and $t_{\mathrm{o}}$ is the desired time of departure from front of queue (time to be on-time).

The cost of schedule delay is thus

$$
\begin{aligned}
& C\left(S_{i}\right)=E *\left|S_{i}\right| \quad \text { if } S_{i}<0 \\
& =L *\left|S_{i}\right| \quad \text { if } S_{i}>0
\end{aligned}
$$

However, we only know the journey delay probabilistically, so we need to solve the schedule delay for each probability of journey delay and add them (we cannot solve the schedule delay for the expected value of journey delay, as on average there may be zero journey delay, but sometimes it is positive and sometimes negative - those do not cancel out but rather are additive). The expected schedule delay cost for traveler $i, E\left(S_{i}\right)$ is determined by the time at which the traveler departs the queue. This is shown in Table 10.

$$
E\left(S_{i}\right)=\sum_{t} P_{t} \Pi_{t}=P(\bar{v}) * 2 E+P(\bar{e}) * E+P(\bar{l}) * L+P(\bar{r}) * 2 L+P(\bar{s}) * 3 L
$$

where $1=\sum_{t} P_{t}=P(\bar{v})+P(\bar{e})+P(\bar{o})+P(\bar{l})+P(\bar{r})+P(\bar{s}) ; P()$ is the probability function for traveler $i$, summarized in Table $10 ; \Pi_{t}=$ penalty function $=(2 E, E, 0, L, 2 L, 3 L) ; \bar{v}, \bar{e}, \bar{o}, \bar{l}, \bar{r}, \bar{s}$ 
Table 10

Departure probability given arrival strategies, three-player game

\begin{tabular}{lllllllllll}
\hline Player B & $v$ & $v$ & $v$ & $v$ & $e$ & $e$ & $e$ & $o$ & $o$ & $l$ \\
Player C & $v$ & $e$ & $o$ & $l$ & $e$ & $o$ & $l$ & $o$ & $l$ & $l$
\end{tabular}

Departure probability

Player A

$v$

$\begin{array}{lllllllllll}P(\bar{v}) & 0.33 & 0.5 & 0.5 & 0.5 & 1 & 1 & 1 & 1 & 1 & 1 \\ P(\bar{e}) & 0.33 & 0.5 & 0.5 & 0.5 & 0 & 0 & 0 & 0 & 0 & 0 \\ P(\bar{o}) & 0.33 & 0 & 0 & 0 & 0 & 0 & 0 & 0 & 0 & 0 \\ P(\bar{l}) & 0 & 0 & 0 & 0 & 0 & 0 & 0 & 0 & 0 & 0 \\ P(\bar{r}) & 0 & 0 & 0 & 0 & 0 & 0 & 0 & 0 & 0 & 0 \\ P(\bar{s}) & 0 & 0 & 0 & 0 & 0 & 0 & 0 & 0 & 0 & 0\end{array}$

$e$

$\begin{array}{lllllllllll}P(\bar{v}) & 0 & 0 & 0 & 0 & 0 & 0 & 0 & 0 & 0 & 0 \\ P(\bar{e}) & 0 & 0.5 & 1 & 1 & 0.33 & 0.5 & 0.5 & 1 & & 1 \\ P(\bar{o}) & 1 & 0.5 & 0 & 0 & 0.33 & 0.5 & 0.5 & 0 & 0 & 0 \\ P(\bar{l}) & 0 & 0 & 0 & 0 & 0.33 & 0 & 0 & 0 & 0 & 0 \\ P(\bar{r}) & 0 & 0 & 0 & 0 & 0 & 0 & 0 & 0 & 0 & 0 \\ P(\bar{s}) & 0 & 0 & 0 & 0 & 0 & 0 & 0 & 0 & 0 & 0\end{array}$

$O$

$\begin{array}{lllllllllll}P(\bar{v}) & 0 & 0 & 0 & 0 & 0 & 0 & 0 & 0 & 0 & 0 \\ P(\bar{e}) & 0 & 0 & 0 & 0 & 0 & 0 & 0 & 0 & 0 & 0 \\ P(\bar{o}) & 1 & 1 & 0.5 & 1 & 0 & 0.5 & 1 & 0.33 & 0.5 & 1 \\ P(\bar{l}) & 0 & 0 & 0.5 & 0 & 1 & 0.5 & 0 & 0.33 & 0.5 & 0 \\ P(\bar{r}) & 0 & 0 & 0 & 0 & 0 & 0 & 0 & 0.33 & 0 & 0 \\ P(\bar{s}) & 0 & 0 & 0 & 0 & 0 & 0 & 0 & 0 & 0 & 0\end{array}$

$l$

\begin{tabular}{lllllllllll}
$P(\bar{v})$ & 0 & 0 & 0 & 0 & 0 & 0 & 0 & 0 & 0 & 0 \\
$P(\bar{e})$ & 0 & 0 & 0 & 0 & 0 & 0 & 0 & 0 & 0 & 0 \\
$P(\bar{o})$ & 0 & 0 & 0 & 0 & 0 & 0 & 0 & 0 & 0 & 0 \\
$P(\bar{l})$ & 1 & 1 & 1 & 0.5 & 1 & 1 & 0.5 & 0 & 0.5 & 0.33 \\
$P(\bar{r})$ & 0 & 0 & 0 & 0.5 & 0 & 0 & 0 & 1 & 0.5 & 0.33 \\
$P(\bar{s})$ & 0 & 0 & 0 & 0 & 0 & 0 & 0 & 0 & 0 & 0.33 \\
\hline
\end{tabular}

are the periods of departure from the queue (very early $\left(t_{\mathrm{o}}-2\right)$, early $\left(t_{\mathrm{o}}-1\right)$, on-time $\left(t_{\mathrm{o}}\right)$, late $\left(t_{\mathrm{o}}+1\right)$, really late $\left(t_{\mathrm{o}}+2\right)$, super late $\left.\left(t_{\mathrm{o}}+3\right)\right)$.

We can characterize the results of the three-player game for different early, delay, and late penalties. The results are shown in Table 11, which for a variety of penalties identifies the number of solutions, and characterizes the solutions according to arrival pattern. We can also look at the impact of marginal cost pricing, which reduces the number of equilibrium solutions to a subset of the unpriced conditions, and achieves the minimum total cost (which only sometimes occurs in unpriced conditions). It should be noted that in certain cases, there are alternative lowest total cost solutions that are not equilibria.

To illustrate the gains from pricing, for instance, in the case: $E=3, D=1, L=4$, the unpriced equilibrium solution eee has a total cost of 10 , compared with the priced equilibria of eoo, oeo, ooe which have a total cost of 7 . 
Table 11

Results from three-player congestion game

\begin{tabular}{|c|c|c|c|c|c|}
\hline$E, D, L$ & $\begin{array}{l}\text { Number of } \\
\text { Nash equilibria } \\
\text { (unpriced) }\end{array}$ & Solutions (Unpriced) & $\begin{array}{l}\text { Number of } \\
\text { Nash equilibria } \\
\text { (priced) }\end{array}$ & Solutions (priced) & $\begin{array}{l}\text { Lowest } \\
\text { total cost }\end{array}$ \\
\hline $0,0,0$ & 64 & all & 64 & all & 0 \\
\hline $0,1,0$ & 24 & veo ...,vel..,vol $\ldots$, eol..., & 24 & veo...,vel...,vol...,eol..., & 0 \\
\hline $0,0,1$ & 13 & vvv,vvo $\ldots, v e e \ldots, v e o, \ldots$ & 13 & vvv,vvo $\ldots$, vee $\ldots, v e o, \ldots$ & 0 \\
\hline $0,1,1$ & 6 & veo,$\ldots$ & 6 & veo, $\ldots$ & 0 \\
\hline $1,0,0$ & 8 & ooo, $l l l$, oll,$\ldots$, ool $\ldots$ & 8 & ooo $, l l l,, l l o, \ldots$, ool $\ldots$ & 0 \\
\hline $1,1,0$ & 7 & ooo, ool $\ldots$, oll,$\ldots$ & 3 & $l l o, \ldots$ & 1 \\
\hline $1,0,1$ & 5 & ооо $, е е е, \ldots$, еео,$\ldots$ & 4 & $е е е, е е о, . .$. & 2 \\
\hline $1,1,1$ & 9 & $e o l, \ldots, e o o, \ldots$ & 6 & $e o l, \ldots$ & 2 \\
\hline $3,1,4$ & 1 & eеe & 3 & $e o o, \ldots$ & 7 \\
\hline $4,0,3$ & 2 & ооо,еее & 4 & еее, еоо & 7 \\
\hline $4,1,3$ & 4 & ооo $, e о o, \ldots$ & 9 & $e o l, \ldots, e o o$ & 7 \\
\hline $3,0,4$ & 1 & eеe & 1 & eee & 7 \\
\hline
\end{tabular}

Note: Column 1 gives the penalty for arriving early, suffering journey delay, or arriving late. The characterization of solutions identifies which patterns are Nash equilibria, $v$ indicates vehicles arrive very early, $e$ early, $o$ on-time, $l$ late.

Sometimes there are more equilibria with pricing, sometimes there are fewer. For instance, in the next to last line of Table 11, where the penalties are $E=4, D=1, L=3$, pricing enables the uncongested strategy eol (and variants) equilibria, but throws out the congested ooo equilibrium, which despite being a stable equilibrium in the unpriced case, had a total cost of 12 compared with a low cost solution of 7. The strategy eol is not an equilibrium without pricing because travelers in the early and late periods can individually do better if the other travelers do not change their decision. (Note: the total costs to users do not include the prices, which are assumed to be returned to travelers in some form, and thus comprise a transfer rather than a cost).

It turns out that, in the cases examined, when there are penalties for late arrivals there are no silently suffering "martyrs", individuals who departed later than others and still suffered journey delay (e.g., the late arriver in case: ool, or the on-time arriver in case eeo). The case ool is only an equilibrium when there is no late penalty. A similar case is eeo. The on-time arriver will depart the queue late, because there is queueing in the early period spilling over. While this is a dysfunctional equilibrium in the unpriced case $(1,1,1)$ (it has a total cost of 3 , compared with a minimum total cost of 2 for scenarios like eol), pricing eliminates this outcome.

\section{Conclusions}

This paper has developed a new way of viewing congestion and congestion pricing in the context of game theory. Simple interactions among players (vehicles) affect the payoffs for other players in a systematic way. Based on the value of time for various activities (time at origin, at destination, or on-the-road), departure times, and consequently congestion, will vary for the players. Under some range of values congestion will occur between non-cooperative players, 
even if both would be better off making a different decision. A prisoners' dilemma is one of many possible outcomes. Congestion is an outcome of individually rational, and sometimes globally rational behavior, under certain preferences. Where there is a difference between the individual and global rational outcomes, congestion pricing can be used, which reduces congestion to an optimal level (but may not eliminate it). The actual early, late, and delay penalties depend on the individual's value of time, and it is easy to extend the model to handle differentiated values of time.

This analysis assumed that players played pure strategies. Extensions to consider mixed strategies (where the play varies from day-to-day with some probability) may yield additional insights.

For certain travelers and certain trips, what is most important is not the amount of additional journey time, but instead the certainty associated with arrival time. The importance of this factor depends on the preference function of travelers and the type of trip. In particular, in many cases journey time is affected by the unknown. The randomness in the system and lack of real-time information lead to travel being stochastic. Risk averse travelers may control for this by departing earlier. The relaxation of the assumption of perfect information is an important extension of the model. The use of pricing as an information signal (that few if any actually pay) may be an important result.

\section{Appendix A}

Eight cases from the basic model are given below.

\begin{tabular}{llll}
\hline Vehicle 1 & Vehicle 2 & Late \\
\cline { 2 - 4 } & Early & On-time & {$[0,0]^{*}$} \\
\hline$E, D, L 0,0,0$ & & & {$[0,0]^{*}$} \\
Early & {$[0,0]^{*}$} & {$[0,0]^{*}$} & {$[0,0]^{*}$} \\
On-time & {$[0,0]^{*}$} & {$[0,0]^{*}$} & \\
Late & {$[0,0]^{*}$} & & {$[0,0]^{*}$} \\
$E, D, L 0,1,0$ & & {$[0,0]^{*}$} & {$[0,0]^{*}$} \\
Early & {$[0.5,0.5]$} & {$[0.5,0.5]$} & {$[0.5,0.5]$} \\
On-time & {$[0,0]^{*}$} & {$[0,0]^{*}$} & \\
Late & {$[0,0]^{*}$} & & {$[0,1]$} \\
$E, D, L 0,0,1$ & & {$[0,0]^{*}$} & {$[0,1]$} \\
Early & {$[0,0]^{*}$} & {$[0.5,0.5]$} & {$[1.5,1.5]$} \\
On-time & {$[0,0]^{*}$} & {$[1,0]$} & {$[0,1]$} \\
Late & {$[1,0]$} & {$[0,0]^{*}$} & {$[0,1]$} \\
$E, D, L 0,1,1$ & & {$[1,1]$} & {$[2,2]$} \\
Early & {$[0.5,0.5]$} & {$[1,0]$} &
\end{tabular}


Appendix A (continued)

\begin{tabular}{llll}
\hline Vehicle 1 & \multicolumn{2}{l}{ Vehicle 2} & \\
\cline { 2 - 4 } & Early & On-time & Late \\
\hline$E, D, L 1,0,0$ & & & \\
Early & {$[0.5,0.5]$} & {$[1,0]$} & {$[1,0]$} \\
On-time & {$[0,1]$} & {$[0,0]^{*}$} & {$[0,0]^{*}$} \\
Late & {$[0,1]$} & {$[0,0]^{*}$} & {$[0,0]^{*}$} \\
$E, D, L 1,1,0$ & & & \\
Early & {$[1,1]$} & {$[1,0]$} & {$[1,0]$} \\
On-time & {$[0,1]$} & {$[0.5,0.5]$} & {$[0,0]^{*}$} \\
Late & {$[0,1]$} & {$[0,0]^{*}$} & {$[0.5,0.5]$} \\
$E, D, L 1,0,1$ & & & \\
Early & {$[0.5,0.5]$} & {$[1,0]$} & {$[1,1]$} \\
On-time & {$[0,1]$} & {$[0.5,0.5]^{*}$} & {$[0,1]$} \\
Late & {$[1,1]$} & {$[1,0]$} & {$[1.5,1.5]$} \\
& & & \\
$E, D, L 1,1,1$ & {$[1,1]$} & {$[1,0]^{*}$} & {$[1,1]$} \\
Early & {$[0,1]^{*}$} & {$[1,1]^{*}$} & {$[0,1]^{*}$} \\
On-time & {$[1,1]$} & {$[1,0]^{*}$} & {$[2,2]$} \\
Late & & & \\
\hline
\end{tabular}

Note: ${ }^{*}$ indicates Nash equilibrium [Vehicle 1 payoff; Vehicle 2 payoff]. Early arrival penalty (E), Delay penalty $(D)$, Late arrival penalty $(L)$.

\section{References}

Arnott, R., de Palma, A., Lindsey, R., 1990. Economics of a bottleneck. Journal of Urban Economics 27, 111-130.

Arnott, R., de Palma, A., Lindsey, R., 1993. A structural model of peak-period congestion: a traffic bottleneck with elastic demand. American Economic Review 83 (1), 161-179.

Arnott, R., de Palma, A., Lindsey, R., 1998. Recent developments in the bottleneck model. In: Button, K.J., Verhoef, E.T. (Eds.), Road Pricing, Traffic Congestion and the Environment: Issues of Efficiency and Social Feasibility. Edward Elgar, Cheltenham.

Axelrod, Robert, 1984. The Evolution of Cooperation. Basic Books, New York.

Hansen, M., Wenbei, W., 2001. An airline's choice of aircraft size in a competitive environment. In: Proceedings Presented at INFORMS Conference Miami, November 2001.

Hargreaves-Heap, Shaun, P., Yanis, V., 1995. Game Theory: a Critical Introduction. Routledge, New York.

Hildebrand, M., Prentice, B., Lipnowski, I., 1990. Enforecement of highway weight regulations: a game theoretic model. Journal of the Transportation Research Forum 30 (2), 442-452.

Jankowski, W.B., 1991. Fare Evasion and non-compliance: a game theoretical approach. International Journal of Transport Economics 38 (3), 275-287.

Kita, H., Tanimoto, K., Fukuyama, K., 2001. An inverse analysis of merging-giveway game: focusing on the equilibrium selection, Presented at the Second Berkeley-Tottori Joint Seminar on Evolution Processes of Transportation Systems: Analyses and Policy Implications, June.

Knight, Frank, 1924. Some fallacies in the interpretation of social cost. Quarterly Journal of Economics 38, 582-606. 
Levinson, D., 1999. Tolling at a frontier: a game theoretic approach. In: Proceedings of the 14th International Symposium on Transportation and Traffic Theory, pp. 173-187.

Levinson, David, 2000. Revenue choice on a serial network. Journal of Transport Economics and Policy 34 (1), 69-98.

Lindsey, C. Robin, Erik T., Verhoef, 2000. Traffic Congestion and Congestion Pricing, TI 2000-101/3, Tinbergen Institute Discussion Paper http://www.tinbergen.nl/discussionpapers/00101.pdf.

Littlechild, S.C., Thompson, G.F., 1977. Aircraft landing fees: a game theory approach. The Bell Journal of Economics 8, 186-203.

Marini, Marco A., Marcucci, E., 2003. Political acceptability of road pricing policies under individual specific uncertainty. In: Schade, J., Schlabe, K. (Eds.), Acceptability of Transport Pricing Strategies. Elsevier Science, pp. 279-297.

Mohring, Herbert, 1970. The peak load problem with increasing returns and pricing constraints. American Economic Review 60, 693-705.

Osborne, Martin J., Rubinstein, Ariel, 1994. A Course in Game Theory. MIT Press, Cambridge MA.

Rapoport, Anatol, 1970. N-Person Game Theory: Concepts and Applications. The University of Michigan Press, Ann Arbor.

Schelling, Thomas C., 1978. Micromotives and Macrobehavior. W.W. Norton and Company.

Small, K.A., 1982. The scheduling of consumer activities: work trips. American Economic Review 72 (3), 467-479.

Taylor, Michael, 1987. The Possibility of Cooperation. Cambridge University Press, Cambridge.

Vickery, W., 1969. Congestion theory and transport investment. American Economic Review 59, 251-260.

Von Neumann, J., Morgenstern, O., 1944. Theory of Games and Economic Behavior. Princeton University Press, Englewood Cliffs, NJ.

Walters, A.A., 1961. The theory and measurement of private and social cost of highway congestion. Econometrica 29, 676-699.

Wardrop, J.G., 1952., Some theoretical aspects of road traffic research. In: Proceedings, Institution of Civil Engineers, Part II, vol. 1, pp. 325-378. 